INFECTIOUS DISEASE

\title{
Urethral macrophages - a new HIV-1 reservoir
}

\section{macrophages \\ are a major \\ HIV-1 reservoir \\ in the penile urethra}

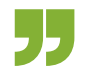

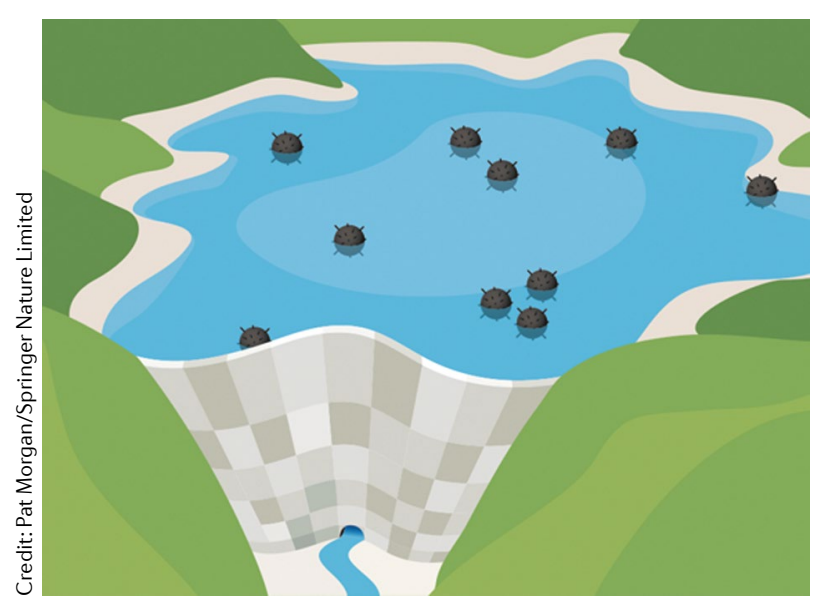

Eradication of latent HIV-1

reservoirs, which are routinely studied in circulating T cells, is a major clinical challenge. However, increasing evidence suggests the existence of other cellular HIV-1 reservoirs, including tissue-resident macrophages. A new study now reports that urethral macrophages are a principal reservoir of replicationcompetent HIV-1 in penile tissues from HIV-1-infected individuals undergoing suppressive combination antiretroviral therapy (cART).

"We previously discovered that, upon entry into the penile urethra, HIV-1 targets urethral macrophages, but not urethral T cells, which led us to investigate whether urethral tissue macrophages could form replication-competent HIV-1 reservoirs," explains lead investigator Yonatan Ganor. Accordingly, the authors evaluated whole penile tissues - recovered during elective gender reassignment surgery - from HIV-1-infected individuals undergoing cART who had undetectable plasma viral loads (HIV-1-cART individuals; $n=20$ ).

The authors first used nested PCR and in situ hybridization to investigate the presence of integrated
HIV-1 DNA in urethral tissues from HIV-1-cART individuals and uninfected controls. Strikingly, HIV1 -specific products were detected in HIV-1-cART, but not control, tissues. Furthermore, integrated HIV-1 DNA was only detected in CD68 ${ }^{+}$ macrophages, but was undetectable in $\mathrm{CD}^{+} \mathrm{T}$ cells, suggesting that urethral macrophages are the main population containing integrated HIV-1 DNA. In addition, HIV-1 RNA was detected in $\mathrm{CD}_{68}{ }^{+}$macrophages but not $\mathrm{CD}^{+}$ T cells from HIV-1-cART urethral tissues, showing that HIV-1 RNA persists in urethral macrophages during suppressive cART.

The investigators also developed a viral outgrowth assay adapted to tissue macrophages that provided a readout of the outgrowth of replication-competent infectious HIV-1 in single-cell suspensions prepared from the epithelial and stromal compartments of HIV-1cART urethral tissues. Treatment with lipopolysaccharide (LPS), a latency-reverting agent (LRA) that specifically reactivates HIV-1 production in macrophages, induced HIV-1 outgrowth. However, HIV-1 outgrowth was not detected following treatment with the $\mathrm{T}$ cell-specific LRA phytohaemagglutinin, suggesting that macrophages, but not T cells, form an HIV-1 reservoir in these tissues. In confirmation, depletion of macrophages using magnetic beads in combined epithelial-stromal cell suspensions almost completely abolished HIV-1 outgrowth on LPS treatment.

In line with DNA and RNA level data, fluorescence microscopy revealed that the HIV-1 capsid protein p24 colocalized with $\mathrm{CD} 4^{+} \mathrm{CD} 68^{+}$ macrophages in the urethral stroma of HIV-1-cART individuals and that p24 was negative in stromal $\mathrm{CD} 68^{-} \mathrm{CD} 4^{+}$
T cells. Further morphological assessment of HIV-1-containing compartments in macrophages using confocal microscopy showed that HIV-1 proteins were only detected within $\mathrm{CD}^{+} 8^{+}$macrophages (and not $\mathrm{CD}^{+} \mathrm{T}$ cells) and were contained only within intracellular structures, inferring HIV-1 compartmentalization within virus-containing compartments (VCCs). Accordingly, using electron microscopy, epithelial and stromal urethral macrophages were shown to harbour intracellular VCC-like vesicular structures that enclosed HIV-1 virions. Finally, multiparametric flow cytometry coupled with immunofluorescence microscopy experiments demonstrated that a unique subset of macrophages with intermediate polarization (termed $\mathrm{Mi}$ ) — which co-express M1 and M2 polarization markers - were enriched in urethral tissues from HIV-1-cART individuals and preferentially contain HIV-1.

In summary, the study reveals that macrophages are a major HIV-1 reservoir in the penile urethra of HIV-1-cART individuals, challenging the dogma that HIV-1 reservoirs principally reside in $\mathrm{T}$ cells.

The findings also have clear relevance for therapeutic HIV-1 eradication strategies "This information is crucial for 'shock and kill' strategies aimed at reservoir elimination. To be effective, such new strategies should also consider, and be adapted to, purging HIV-1 from reservoirs in macrophages," adds Ganor. "For instance, we are investigating whether LPS-TLR4 signalling and clinically approved TLR4 agonists could be exploited to reactivate this newly identified macrophage reservoir, in order to achieve the clinical goal of HIV-1 eradication."

Conor A. Bradley

ORIGINAL ARTICLE Ganor, Y. et al. HIV-1 reservoirs in urethral macrophages of patients under suppressive antiretroviral therapy. Nat. Microbiol. https://doi.org/10.1038/s41564-018-0335-z (2019) 